\title{
Cultura organizacional desde la teoría de Edgar Schein: Estudio fenomenológico
}

\author{
Organizational culture Edgar Schein's theory: A phenomenological study
}

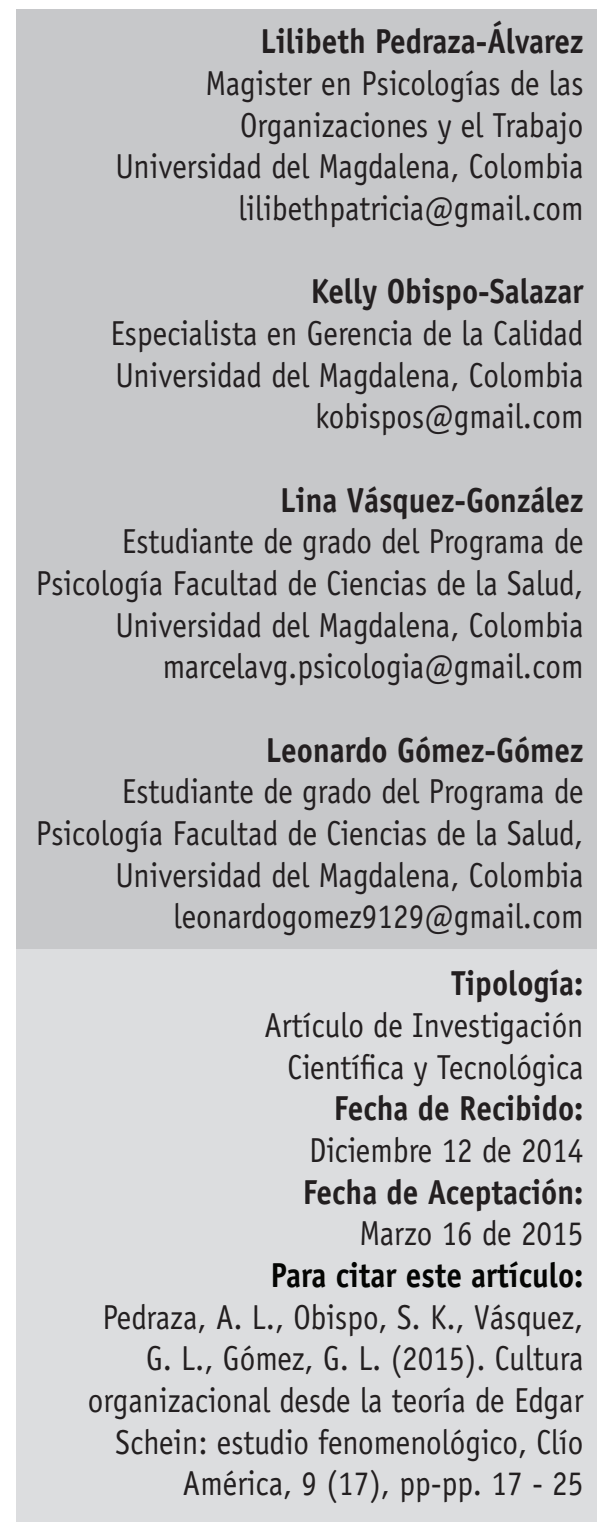

Resumen: En el siguiente trabajo se presenta el estudio de un fenómeno de la vida corporativa haciendo una descripción de la cultura organizacional en tres instituciones de carácter educativo en la ciudad de Santa Marta-Colombia, cuyo objetivo está encaminado a describir la cultura organizacional, desde donde es posible evidenciar distintas dinámicas como parte del funcionamiento y las relaciones en términos del contexto en el que se desarrollan las instituciones. Este es un estudio cualitativo, bajo la perspectiva metodológica de la fenomenología, de corte transversal, con una muestra de 30 personas, (docentes y personal del cuerpo administrativo), seleccionado a partir de un muestreo por conveniencia. Se recolectó la información a partir del uso de la técnica de los grupos focales, y como instrumento de recolección de información, la entrevista semiestructurada. Se encontró que estas instituciones se encuentran dentro de un nivel de cultura elemental (Nivel 1), lo cual es producto, de acuerdo con Schein (1988), a la falta de integración y la introducción de las presunciones primordiales, en un grado básico de estabilidad frente a la consecución de soluciones a varias alternativas.

Palabras clave: Cultura; organizaciones; presunciones básicas; relaciones; niveles de cultura.

\section{JEL: Z130}

Abstract: The article presents the study of a phenomenon of corporate life from the description of the organizational culture in three organizations of educational character in Santa Marta-Colombia, whose objective was guided to describe the organizational culture, where it is possible to show different dynamics as part of the operation and relationships in terms of the context in which institutions develop. This is a qualitative study on the methodological perspective of phenomenology, transversal, with a sample of 30 people (teachers and administrative staff of the body), selected from a convenience sample. Information from the use of the technique of focus groups was collected, and an instrument of information gathering semistructured interview. It was found that; these institutions are within a culture elemental level (Level 1) which is the results to, according to Schein (1988), the lack of integration and the introduction of basic presumptions at a basic level of stability achieve solutions to several basic alternatives.

Keywords: Culture; organizations; basic assumptions; relationships; cultural level. 


\section{Introducción}

Al momento de hablar de cultura organizacional es necesario tener en cuenta las dinámicas funcionales referidas a aspectos administrativos y relacionales en el grupo de trabajo, ya que la industria no se puede definir únicamente desde el punto de vista de un empleado, como tampoco se puede definir a partir desde el punto de vista de una empresa particular, es por tanto que dependen de la relación jerárquica y la subordinación (Abravanel, Allaire, Firsirotu, Hobbs, Poupart, Simard, 1992).

“Dado que la cultura organizacional se constituye con percepciones del mundo y productos simbólicos, entonces llega a ser ésta un contexto dinámico cargado de símbolos, cogniciones funcionales o una estructura mental profunda y subconsciente" (Abravanel et al, 1992, p.15), es por tanto que se hace necesario acudir al discurso de quienes participan de esta investigación. Por lo anterior, el objetivo de este trabajo fue la descripción de la cultura organizacional, dado que permitió evidenciar la manera en que se manifiestan estas dinámicas, teniendo en cuenta las exposiciones de los participantes, pertenecientes a un grupo de instituciones educativas del distrito de Santa Marta, correspondiente al cuerpo docente y administrativo.

De acuerdo al planteamiento expuesto, surgen interrogantes en relación a la necesidad de considerar la cultura organizacional como un factor determinante en la eficacia del personal que labora en cada una de las Instituciones Educativas (Valverde y Valverde, 2001).

Así se entiende que existen manifestaciones internas de la cultura organizacional, dentro de los cuales encontramos, según lo planteado por Schein (1988), el “lenguaje común y categorías conceptuales, limites grupales y criterios para la inclusión y exclusión, poder y jerarquía, intimidad, amistad y amor, recompensas y castigos, ideología y religión" (p. 79). Aspectos que facilitan a las entidades anticipar y adoptar los cambios que le plantea el entorno para mejorar su servicio y satisfacer con calidad las demandas que le impone la sociedad. Justamente es cómo se pretende comprender, identificar y reconocer el nivel en el que se encuentra la cultura organizacional de estas instituciones de acuerdo a lo planteado por Schein en 1988.

\section{Definición de cultura}

La cultura organizacional es una temática que ha tenido gran relevancia e importancia aún desde los años 70 u 80; es necesario que la cultura organizacional esté presente en cada organización, ya que ésta es la encargada de medir el rendimiento tanto individual como colectivo de los trabajadores(Robbins, 2005, citado por Miquilena y Paz, 2008).

Con el transcurrir del tiempo han surgido cambios en los diferentes sistemas, ya sea en lo educativo, en lo social, en lo individual y en lo organizacional. En este último campo, se ha generado en el mundo un alto impacto, puesto que, es el órgano que indica producción y crecimiento. Como lo plantea Lozada (2004) reseñando los planteamientos de Marín y García (2002), si bien las organizaciones son producto de un desarrollo histórico en donde sus características han dependido de todo un recorrido evolutivo desde la manufactura hasta los más grandes sucesos de la industrialización, es muy importante destacar que las organizaciones tienen como base la coherencia, pero sobre todo los aportes que cada individuo hace a las mismas, teniendo en cuenta sus costumbres, ideales y demás aspectos que influyen en sus pensamientos y más aún los avances de la ciencia (Marín y García, 2002, citado por Lozada, 2004).

Schein, en 1988 introdujo el concepto de presunciones y creencias, para explicar de forma más amplia el significado que para la organización tienen el concepto de cultura, la define entones como "... respuestas que ha aprendido el grupo ante sus problemas de subsistencia en su medio externo y ante sus problemas de integración interna" (Schein, 1988, p. 24), este nivel de presunciones y creencias que comparten los grupos en la organización corresponde a la esencia misma de la cultura. La cultura debe ser vista como el conjunto de experiencias importantes y significativas tanto internas y externas que los individuos en una empresa han experimentado e implementado estrategias para adaptarse a la organización. Producto de esto se ha generado una vivencia común de lo que los rodea 
y el lugar que ocupan dentro de la organización. Como sugiere Schein (1988) "en un concepto formal para la cultura organizacional, en tal sentido es un producto aprendido de la experiencia, y por tanto algo localizable solo allí, donde exista un grupo definible y poseedor de una historia significativa..." (pp. 24-25).

\section{Aproximación a las categorías de la cultura organizacional}

Según Schein (1988) en la esencia de la cultura de las organizaciones, se reserva el nivel más profundo de presunciones básicas y creencias (Nivel 3), que permiten a los individuos cada día expresar y experimentar sus acontecimientos, dar respuesta a sus problemas de subsistencia interna y externa. Estas presunciones básicas, son la esencia, lo que realmente es la cultura organizacional, un modelo desarrollado por un grupo para ir aprendiendo a enfrentarse con sus problemas de adaptación. Los valores y conductas desde sus producciones y creaciones, son en efecto manifestaciones derivadas de la esencia cultural.

En 1988, Schein los conceptualiza como niveles de cultura, estos tres niveles de cultura no son estáticos ni independientes, se interrelacionan y conforman las creencias y presunciones básicas de la cultura organizacional:

“El nivel 1: Producciones...está dado por su entorno físico y social. En este nivel cabe observar el espacio físico, la capacidad tecnológica del grupo, su lenguaje escrito y hablado y la conducta expresa de sus miembros.

El nivel 2: Valores, que reflejan en última instancia la manera en que deben relacionarse los individuos, ejercer el poder y pueden ser validados si se comprueba que reducen la incertidumbre y la ansiedad.

El nivel 3: presunciones subyacentes básicas, permite la solución a un problema cuando esta se ha dado repetidamente y queda a la larga asentada..." (Schein, 1988, pp. 30-32).
“Esta perspectiva se resume señalando que la cultura de una organización puede ser estudiada en estos tres niveles, pero, si no se descifra el patrón de supuestos básicos que giran en torno a la organización, no se sabrá cómo interpretar los demás aspectos correctamente; una vez que se comprenden los supuestos básicos, se puede comprender fácilmente los otros niveles que son más superficiales" (Schein, 1992, citado por Soria, 2008, p.26).

Además, permite la integración interna en el afianzamiento de sus procesos de adaptación, para funcionar como un sistema social. Frente a esta función, Schein (1988), plantea que deben existir cuestiones internas que toda empresa debe desarrollar:

“Lenguaje común y categorías conceptuales, si el grupo no puede comunicarse ni entenderse bien, el grupo es imposible. Limites, grupales y criterios para la inclusión y exclusión, conceso común sobre quienes están dentro y quienes fuera. Poder y jerarquía, es vital para que los miembros puedan controlar sus sentimientos agresivos. Intimidad amistad y amor, Relaciones entre iguales y entre los sexos. Recompensas y castigos, todo grupo debe saber cuáles son. Ideología y religión" (Schein, 1988, p. 79).

Con todo esto, es importante tener en cuenta que si bien las grandes potencias económicas en el mundo están incluidas por su contexto, existen elementos dentro de cada organización que las hacen más eficaces que otras y es allí donde el aporte individual y las relaciones de los miembros de una compañía influyen en esta.

\section{Metodología}

Para el logro del objetivo planteado, se hizo sumamente importante la participación activa de las organizaciones (Instituciones Educativas pertenecientes al distrito de Santa Marta), brindando los recursos y apoyos necesarios para cumplir satisfactoriamente con el proceso, puesto que situaciones contrarias o desfavorables terminarían por obstaculizar dicha intervención. 


\section{Tipo de investigación}

El tipo de investigación corresponde al paradigma de investigación cualitativo, donde según Mayan (2001), esta se usa para comprender con naturalidad los fenómenos que ocurren, y tratar de darle sentido a lo que observa en la vida del individuo, sin interrumpirla en su esencia o hacer predicciones acerca de ella, por lo cual el investigador no intenta manipular el escenario de la investigación al controlar influencias externas 0 en el diseño de experimentos, se centra en descubrir fenómenos de los cuales se tiene poca información, y capturar su significado como proceso y no como producto.

El diseño de investigación es fenomenológico el cual implica, como sugiere López (2007), abarcar los subsistemas, la cultura de determinado sistema social y transformar la experiencia vivida así mismo: “consiste precisamente en comprender desde la experiencia misma lo que se revela en ella, no desde una actitud meramente interpretativa, que busque la coherencia interna del texto analizado, sino que aboga por un respeto pleno del relato que hace el hablante, donde expresa la forma como él ha vivido lo que ha vivido" (López, 2007, p. 95).

\section{Población y muestra}

La población total estuvo integrada por los docentes de la comunidad educativa y personal del área administrativa, con un total de 61 sujetos. La muestra fue seleccionada a partir del muestreo por conveniencia, en donde se identificaron los participantes clave para el proceso investigativo teniendo en cuenta los casos disponibles a los cuales se tenía acceso (Hernández, Fernández, Baptista, 2006). A partir de lo anterior se inicia el proceso de recolección de información, con un total de 30 participantes.

\section{Técnicas para la recolección de la información}

Como técnica de recolección de información se usó el grupo focal, que constituye una técnica cualitativa de recolección de información asentada en entrevistas colectivas y semiestructuradas. Los grupos focales apuntan al análisis e interpretación de los comportamientos y prácticas sociales comunes, que implican el observar. Estos no buscan informar sobre la extensión o la cantidad de los mismos, sino que implican dar una explicación basada en las interpretaciones de acuerdo al comportamiento de estos fenómenos (Fontas, Conçalves, Vítale y Viglietta, sf).

Se utilizó también la entrevista semiestructurada caracterizada según Martínez (2006), como la forma que adopta un diálogo corriente y brinda las posibilidades y la significación del diálogo como método de conocimiento de los seres humanos, sobre todo, en la calidad del proceso en el que se apoya, la escucha. Para el análisis de los datos se hizo uso del software Atlas $\mathrm{Ti}$, este programa permite llevar a cabo la identificación de códigos y la formación de las primeras categorías de análisis, lo que permite alcanzar nivel inicial de exploración de todos los documentos escritos producto de las entrevistas para el estudio de datos cualitativos (Hernández et al, 2006). Para el diagnóstico de la cultura de estas organizaciones se realizó un estudio en un periodo de un mes aproximadamente, con ayuda de la planta institucional de las I.E.D., en la ciudad de Santa Marta, correspondiente a docentes y cuerpo administrativo.

\section{Resultados}

Acorde con los métodos empleados, se procedió a estructurar la información de acuerdo a dos grandes categorías: Manejo Administrativo (manejo administrativo y rutas organizacionales), y Relaciones y Ambiente (como se tornan las relaciones entre el cuerpo de trabajo), teniendo en cuenta el discurso de los participantes.

Paralelo con las respuestas de los sujetos por cada Institución Educativa, se presentan los códigos correspondientes a cada una de estas dos categorías, que permiten describir la cultura característica de cada Institución. Seguido se presentan algunos ejemplos narrativos de los comentarios más significativos expresados por el grupo de estudio.

En la categoría manejo administrativo se encontraron los siguientes códigos: control administrativo, desvinculación de procesos, en relación a estudiantes, 
fallas administrativas y formación docente; en la categoría correspondiente a relaciones y ambiente se asignaron los siguientes códigos: buenas relaciones, malas relaciones, trabajo en equipo, problemas de comunicación e infraestructura.

\section{Institución 1}

\section{Manejo Administrativo}

En relación con esta categoría se evidenció que existe una marcada desvinculación de los procesos, recurrentes fallas administrativos, como se evidencia por los participantes.

Con relación a la desvinculación de los procesos se evidenció que: "Cada quien se mete en su trabajo para no tener que hacer nada que no sea benéfico" (Sujeto 2), al mismo tiempo expresa, "La creencia es que los procesos y las iniciativas no se pueden llevar a cabo, y los trabajos que se realizan son producto del trabajo de cada quien" (Sujeto 4).

Los participantes evidenciaron fallas administrativas pues manifestaron: "Los directivos no nos ayudan cuando lo necesitamos" (Sujeto 7).

\section{Relaciones y ambiente}

En esta categoría, la institución se caracterizó por la presencia de buenas relaciones, el trabajo en equipo e infraestructura insuficiente.

Teniendo en cuenta las buenas relaciones, la población hizo alusión a que: "Nosotros nos llevamos bien con el rector y con los coordinadores pero como personas..." (Sujeto 1) Asimismo agregaron, "Entre nosotros los profesores, las relaciones son buenas" (Sujeto 6).

Hicieron referencia al Trabajo en equipo, mencionando que; "Las fortalezas (...) entre nosotros nos ayudamos para enfrentar las problemáticas" (Sujeto 5); además agregan "Los valores se manifiestan en el esfuerzo por parte de los docentes en sacar adelante la institución" (Sujeto 9).
De otro lado, con relación a la infraestructura, consideraron que "Las condiciones del colegio son muy malas, los salones se caen cada vez más, hay huecos en ellos, y son muy pequeños" (sujeto 3).

\section{Institución 2}

\section{Manejo Administrativo}

En esta institución el manejo de las funciones administrativas y la eficacia de los mismos está representada por: fallas administrativas, desvinculación de los procesos, control administrativo, en relación a los estudiantes y formación docente, y los cuales se hacen evidentes así:

En cuanto a las Fallas administrativas consideran que, "Hay poca responsabilidad de los directivos y a nosotros nos toca hacer las cosas de iniciativa propia como creamos (Sujeto 2). Reconocen que, "Hay fallas en los procesos administrativos porque no contamos con un control eficiente de las jornadas de trabajo y se han presentado muchos cambios de asignación de horarios lo que ha dificultado la labor de nosotros (sujeto 1).

De otro lado atendiendo a la Desvinculación de los procesos, manifiestan que "Cada quien hace solo lo que le toca" (Sujeto 8).

Así mismo, en cuanto a Control administrativo argumentan que "Aquí no hay un control de que debe hacer cada quien, se confunden los roles entre uno y otro" (Sujeto 4).

En relación a los estudiantes, exponen que "Nosotros somos estrictos con los alumnos, exigimos que sean disciplinados en el salón y afuera" (Sujeto 3); explican que "Cuando los estudiantes presentan mal comportamiento y van mal académicamente, lo que hacemos es citar a los papas" (Sujeto 5).

Finalmente encontramos que entorno a la Formación docente los participantes expresaron que "Se realizan algunas veces capacitaciones a los docentes, creemos que es importante para que mejoren" (Sujeto 7). 


\section{Relaciones y ambiente}

Esta categoría, está representada por los códigos de infraestructura, trabajo en equipo, problemas de comunicación y buenas y malas relaciones.

Se evidencia por los participantes en relación con la infraestructura que "El colegio no cuenta con las condiciones adecuadas para una grata permanencia en los salones de clase, dada la poca ventilación y falte de servicio de luz" (Sujeto 5).

En términos de Trabajo en equipo, los participantes expusieron que, "Los hábitos (...) están relacionados con mantener un ambiente de trabajo agradable, amistoso y de cooperación" (Sujeto 2); además "Tomamos decisiones en conjunto" (Sujeto 9).

Sin embargo, lo anterior no se percibe óptimamente pues manifiestan Problemas de comunicación, puesto que, "No hay un buen puente de comunicación dado que se han presentado cambios de decisiones a último momento y no se han tenido en cuenta muchas sugerencias por parte de los docentes en cuanto a la formas de evaluación, seguimiento y admisión de estudiantes" (Sujeto 10).

Como consecuencia, los participantes manifiestan Malas relaciones aludiendo que "Existen malas relaciones en el trato con el grupo de secretarias" (Sujeto 8). Sin embargo exponen un sustento de Buenas relaciones entre otros miembros "Los docentes trabajamos bajo el respeto y la tolerancia en el grupo de trabajo" (Sujeto 3).

\section{Institución 3}

\section{Manejo Administrativo}

Esta categoría está representada por los siguientes códigos: en relación a los estudiantes, control administrativo y fallas administrativas, como se observa en las afirmaciones de algunos de los participantes. Es relevante destacar en el siguiente orden estos códigos pues se hace referencia a lo más importante de su sentir. En relación a estudiantes consideran que "Al entrar saludo a los estudiantes, hago una oración, paso la lista, dicto la clase y hago un paréntesis para interactuar con los alumnos sobre sus vidas personales" (Sujeto 6). Así mismo otro participante mencionó, "Saludo, interactúo con ellos sobre sus vidas y con los de los últimos grados les pregunto sobre sus aspiraciones con sus estudios y metas" (Sujeto 5).

De otro lado con respecto al Control administrativo se hace mención de algunas actividades como "Reviso el colegio, miro si no hay anomalias y si las hay, las informo a mis superiores" (Sujeto 2).Así mismo expresan "Nosotros molestamos y compartimos informes de clases. Quien va mal, quien va bien" (Sujeto 1).

Desde otra perspectiva, los participantes manifiestan Fallas administrativas, y sugieren que "El rector es comunicativo pero en coordinación no llegan los casos... hay una tendencia a volarse el conducto regular de las cosas y no solo los estudiantes, también los docentes suelen hacerlo" (Sujeto 4).

\section{Relaciones y Ambiente}

En esta se encuentran aspectos como: condiciones físicas, relaciones interpersonales adecuadas y problemas de comunicación; algunos de los participantes al referirse a la infraestructura explican que "Dentro de las debilidades, pues está el hacinamiento por espacio reducido" (Sujeto 2). Además "Hay poco espacio y eso no da orden a las actividades y eventos esporádicos" (Sujeto 7).

Sin embargo expresan que existen buenas relaciones, puesto que "Nos reunimos, molestamos entre nosotros, nos ponemos al día con las novedades, en recreo también nos reunimos. Todo esto lo hacemos como para desestresarnos" (Sujeto 8). También agregaron "Hay una tendencia a romper ideas de humor. No hay disputas y se trata de mantener eso" (Sujeto 1).

No todo es percibido armónicamente, pues existen Problemas de comunicación evidenciadas en comentarios como "La coordinación no da respuesta a los casos y siempre está lleno. Rectoría da más apoyo a estos y quizá por ello es que los casos llegan directamente a rectoría" (Sujeto 3). A lo anterior añaden que "Falta de comunicación, (...). Hay déficits en canales de comunicación, secretaria específicamente" (Sujeto 5). 
A continuación se presenta la tabla que condensa las categorías de análisis y los códigos correspondientes a cada una de las categorías con base en el análisis de lo expresado por los participantes (Tabla 1).

\section{TTabla 1.}

\section{Categorías y códigos}

\begin{tabular}{|c|c|}
\hline $\begin{array}{c}\text { CATEGORIAS DE } \\
\text { ANÁLISIS }\end{array}$ & Códigos \\
\hline \multirow{2}{*}{$\begin{array}{c}\text { Manejo } \\
\text { administrativo }\end{array}$} & $\begin{array}{c}\text { Control administrativo } \\
\text { Desvinculación de procesos } \\
\text { En relación a estudiantes }\end{array}$ \\
\hline & Fallas administrativas \\
\hline \multirow{2}{*}{ Relaciones y } & Formación docente \\
\hline ambiente & Buenas relaciones \\
\hline & Malas relaciones \\
\hline & Trabajo en equipo \\
\hline & Problemas de comunicación \\
\hline
\end{tabular}

Fuente: información recolectada por los autores en la investigación.

\section{Discusión}

A partir de los resultados encontrados se pudo identificar los elementos significativos que hacen parte de la cultura organizacional en las tres instituciones estudiadas. De esta manera, las categorías y códigos, permiten clasificar las instituciones, teniendo en cuenta los niveles de análisis de la cultura en las organizaciones, propuesto por Schein (1988), así se presenta el análisis de las instituciones sobre estos niveles.

De acuerdo a la información recolectada y las categorías formuladas, se evidencian algunas condiciones en común entre las tres instituciones. Tales como difíciles condiciones físicas, como se observa "no contamos con las condiciones adecuadas para una grata permanencia en los salones de clase, hay poca ventilación y falta de servicio de luz" (Sujeto 1- institución 2).
Además se encontró una marcada coincidencia en las tres instituciones relacionada con un inadecuado manejo administrativo de acuerdo a lo expresado, "El área directiva no se vincula a la llamada de los profesores" (Sujeto 8 - Institución 1).

Por otra parte los participantes manifestaron adecuadas relaciones interpersonales, siendo más evidente en las instituciones 2 y 3, así como se manifiesta "Hay buena relación con el personal operativo. Somos como una familia y nos damos apoyo entre colegas y demás"; Nosotros molestamos y compartimos informes de clases" (Sujeto 4-institución 3). Sin embargo no llegan a ser conductas que generen aprendizaje en la resolución de conflictos 0 instauración de formas adecuadas de resolver las situaciones y que se expresan en comentarios como "adaptarse ante las situaciones y optar solo por hacer nuestro trabajo sin vernos afectados por agentes externos" (Sujeto 4-Institución 1).

El análisis de toda la información lleva a ubicar a estas instituciones en un nivel 1 en su cultura organizacional, ya que los comportamientos adoptados no propenden por una solución, por lo cual, al no servirse de una estrategia en la resolución de distintas problemáticas asentado por un valor, no alcanza un nivel de presunciones básicas. Sin embargo, los aportes que cada individuo hace a la organización e influyen en sus pensamientos de acuerdo con Marín y García (2002), citado por Lozada (2004) aunque se mantienen en un nivel básico y general.

\section{Conclusión}

Se evidenciaron dos aspectos que configuran y llegan a describir la cultura en estas organizaciones educativas; por un lado: Manejo Administrativo, que estarían relacionadas con condiciones como, fallas administrativas, control administrativo, formación docente, desvinculación de los procesos, en relación a estudiantes, y por otro lado, Relaciones y Ambiente, que contienen aspectos dentro de los cuales se encuentran distintas dinámicas en las relaciones interpersonales, trabajo en equipo, problemas de comunicación, y condiciones físicas; se observa a partir de los resultados obtenidos; que estas instituciones, se encuentran dentro de un nivel de cultura elemental, lo cual es producto según Schein (1988), a la falta de unificación e introducción de 
un grado básico de estabilidad que dé respuestas a sus problemas de subsistencia, problemas de unificación interna y obtención de soluciones frente a varias alternativas básicas, aspectos que se logran cuando más personas coexisten para cooperar entre sí y alcanzar metas comunes y que no pueden ser alcanzadas individualmente, en la creación de una adecuada cultura (Chiavenato, 1994, citado por Valverde y Valverde, 2001).

Siendo más precisos, es válido afirmar que la falta de integración de la esencia de la cultura organizacional, las presunciones básicas en las Instituciones Educativas estudiadas, no permite comprender fácilmente los niveles 1 y 2, por lo cual tampoco se puede llegar al elemento que genera dichas manifestaciones, es decir las presunciones básicas, el nivel 3; esto pone de manifestó lo esbozado antes por Schein al afirmar que estos tres niveles no son estáticos ni independientes sino que se interrelacionan y conforman las creencias y presunciones básicas de la cultura organizacional.

A partir del estudio realizado, se pudo reconocer que es necesario explorar la cultura en las organizaciones ya que permite determinar hasta qué punto la integración de estos niveles de observación ayudan en la creación de un nivel de cultura más elevado, aspectos que sirven a través de esta investigación como punto de partida en la observación y estudio de la cultura organizacional. Los planteamientos de Schein (1988), permiten ahondar en la importancia que tiene para la organización de la cultura como fenómeno que reconoce tanto la integración de los miembros organizativos y de igual forma, su importante función en el ajuste externo frente a un entorno cambiante, teniendo en cuenta que todo sistema debe seguir un ciclo guía para hacer frente a problemas y cuestiones de adaptación.

\section{Referencias Bibliográficas}

Abravanel, H; Allaire, Y; Firsirotu, M; Hobbs, B; Poupart, R; Simard, J. (1992). Cultura organizacional: aspectos teóricos, prácticos y metodológicos. Leggis. Bogotá.

Carrillo, W. (2010).implementación de un modelo de comunicación interna para apoyar la fidelización del personal con la empresa: Caso indutexma productora de hilos y telas en la ciudad de Otavalo. Recuperado de http://repositorio. ute.edu.ec/bitstream/123456789/8255/1/43330_1.pdf
Checalla, J. (2013). Implementación de la cultura organizacional. Recuperado de http://www.monografias.com/ trabajos95/implementacion-cultura-organizacional/ implementacion-cultura-organizacional.shtml

Fontas, C; Conçalves, F; Vítale, C; Viglietta, D. (sf). La técnica de los grupos focales en el marco de la investigación socio - cualitativa Recuperado de http://www.fhumyar.unr.edu. ar/escuelas $/ 3 /$ materiales $\% 20$ de $\% 20$ catedras/trabajo $\% 20$ de\%20campo/profesoras.htm

González, F. (2006). Investigación cualitativa y subjetividad. Recuperado de http://www.odhag.org.gt/ pdf/R_INVESTIGACION\%20CUALITATIVA.pdf

Hernández, R; Fernández, C. y Baptista, P. (2006). Metodología De la Investigación. McGillUniversity.

Husserl, E. (1962). Ideas relativas a una fenomenología pura y una filosofía fenomenológica. Recuperado de http:// profesorvargasguillen.files.wordpress.com/2012/11/ husserl-edmund-ideas-relativas-a-una-fenomenologiapura-y-una-filosofia-fenomenologica-ocr.pdf

López, H. (2007). Investigación cualitativa y participativa. Universidad Pontificia Bolivariana. Recuperado de http://eav.upb.edu.co/banco/sites/default/files/files/ INVESTIGACIONPSICOLOGIAYEDUCACIONAMBIENTAL_0.pdf

Lozada, N. (2004). Sociología de las organizaciones, de Antonio Lucas Marín y Pablo García Ruiz. Año 19 núm. 54. P. 283288. Recuperado de http://www.revistasociologica.com. $\mathrm{mx} / \mathrm{pdf} / 5414 . \mathrm{pdf}$

Martínez, M. (2006). La investigación cualitativa (síntesis conceptual). Revista IIPSI Vol. 9 N$^{\circ} 1$. Recuperado de http://sisbib.unmsm.edu.pe/bvrevistas/investigacion_psicologia/v09_n1/pdf/a09v9n1.pdf

Mendoza, R. (2006). Investigación cualitativa y cuantitati$v a$ - Diferencias y limitaciones. Recuperado de http:// oportunidades.gob.mx/Portal/work/sites/Web/resources/ ArchivoContent/1351/Investigacion_cualitativa_y_cuantitativa.pdf

Miquilena, D; Paz, A. (2008). La cultura como fuente de compromiso del capital humano en las organizaciones modernas. Recuperado de http://www.publicaciones.urbe.edu/index. php/forumhumanes/article/viewArticle/475/1170.

Mayan, M. (2001). Una introducción a los métodos cualitativos: Modulo de entrenamiento para estudiantes y profesionales. Recuperado de http://www.ualberta.ca/ iiqm/pdfs/ introduccion.pdf

Reichardt, A; Urdaneta, G. (2006). Clima organizacional en el hospital de especialidades pediátricas. Recuperado de http://200.35.84.131/portal/bases/marc/texto/3201-06-00340.pdf 
Rodríguez, G; Gil, J \& García, E. (1996). Metodología de la investigación cualitativa. Recuperado de http://metodosdeinvestigacioninterdisciplinaria.bligoo.com.co/media/users/10/528344/files/53953/ INVESTIGACION_CUALITATIVA_Rodriguez_et_al.pdf

Sandoval, C. (2002). Investigación cualitativa. Recuperado de http://epistemologia-doctoradounermb.bligoo.es/ media/users/16/812365/files/142090/INVESTIGACION_ CUALITATIVA.pdf

Salamanca, A \& M, C.- (sf). El diseño en la investigación cualitativa. Nure Investigación $N^{\circ} 26$. Recuperado de http:// www.nureinvestigacion.es/ficheros_administrador/f_metodologica/fmetodologica_26.pdf

Schein, E. (1988). La cultura empresarial y el liderazgo. Una visión dinámica. Plaza \& Janes Editores. P.
Soria, R. (2008). Emprendurismo, cultura, clima y comunicación organizacional y su aplicación a la pequeña y mediana empresa en la zona metropolitana de Guadalajara, México. Recuperado de http://www.eumed.net/librosgratis/2008c/432/La\%20cultura\%20segun\%20Schein.htm Valda, J. (2011). Cultura organizacional, Liderazgo y habilidades personales. Recuperado de https://jcvalda.wordpress.com/2011/02/26/ cultura-organizacional-liderazgo-y-habilidades-personales/ Valverde, A Y Valverde, S. (2001). La cultura y el clima organizacional como factores relevantes en la eficacia del instituto de oftalmología. Recuperado de http://sisbib.unmsm.edu.pe/ bibvirtualdata/Tesis/Human/Alvarez_V_S/t_completo.pdf 